\title{
Effective Higgs-quark-quark couplings from a heavy supersymmetric spectrum
}

\author{
Antonio Dobado* \\ Departamento de Física Teórica, Universidad Complutense de Madrid, 28040 Madrid, Spain \\ María J. Herrero ${ }^{\dagger}$ and David Temes \\ Departamento de Física Teórica, Universidad Autónoma de Madrid, Cantoblanco, 28049 Madrid, Spain
}

(Received 24 July 2001; published 3 April 2002)

\begin{abstract}
In this paper we study the Yukawa Higgs-quark-quark interactions that are generated from radiative corrections of squarks and gluinos, in the minimal supersymmetric standard model. We compute the corrections to the effective action for Higgs and quark fields that are produced by explicit integration in the path integral formalism of all the squarks and gluinos at the one-loop level and order $\alpha_{s}$. In addition, we consider the limit of nearly degenerate heavy squarks and gluinos, with masses much larger than the electroweak scale, and we derive the effective Lagrangian containing all the relevant new local Higgs-quark-quark interactions. We show that these new interactions do remain nonvanishing, even in the case of infinitely heavy supersymmetric particles and, therefore, we demonstrate explicitly the nondecoupling behavior of squarks and gluinos in Higgs bosons physics. We present the set of new Yukawa couplings and finally derive the corresponding one-loop, order $\alpha_{s}$, corrections to the Higgs bosons partial decay widths into quarks.
\end{abstract}

DOI: $10.1103 /$ PhysRevD.65.075023

PACS number(s): 14.80.Cp

\section{INTRODUCTION}

The Higgs particle discovery and the subsequent study of its physical parameters and couplings is probably the most urgent challenge for particle physics phenomenology in the near future. The major task of this analysis, once one or several Higgs particles have been discovered, will be to unravel their supersymmetric (SUSY) or nonsupersymmetric origin. Obviously, the best evidence for the existence of supersymmetry in nature will be the discovery of genuine SUSY particles, but it may well happen that the SUSY spectrum turns out to be too heavy to be produced directly in the planned experiments. In this case, and in order to prove the existence of SUSY particles, it will be mandatory to search for indirect SUSY signals via their contribution to the radiative corrections in either couplings, physical parameters, as particle masses and/or decay widths, or precision observables, in a similar way as it has been done in the past at the CERN $e^{+} e^{-}$collider LEP for indirect searches of the top quark and the standard model (SM) Higgs particle. In fact, there is already an extensive work done on these SUSY radiative corrections and their phenomenological implications in the literature [1].

In this work we study the radiative corrections from heavy SUSY particles to the Yukawa Higgs-quark-quark interactions using a different but powerful approach provided by the effective action formalism. We concentrate here on the contributions from the SUSY-QCD sector of the minimal supersymmetric standard model (MSSM) but extensions of this work to the SUSY-electroweak sector of the MSSM and to other SUSY models can be easily performed. In short, the procedure goes as follows. We start with the tree-level

\footnotetext{
*Email address: malcon@ fis.ucm.es

${ }^{\dagger}$ Email address: herrero@delta.ft.uam.es

${ }^{\ddagger}$ Email address: temes@delta.ft.uam.es
}

MSSM Lagrangian [2,3], and consider the Higgs sector interactions with quarks and the relevant SUSY-QCD sector interactions. These are the Higgs-squark-squark interactions and the squark-quark-gluino interactions. We perform a standard functional integration, to one-loop level, of all the squarks and gluinos and get the effective action containing all the exact, non-local, one-loop level interactions of the MSSM Higgs particles with quarks. We next examine the limit where the SUSY particles are much heavier than the electroweak scale, $m_{E W} \sim \mathcal{O}(246 \mathrm{GeV})$, and find out the effective local interactions of these Higgs particles with quarks being generated at one-loop level and at low energies from the heavy SUSY-QCD spectrum. These effective local interactions come, in our approach, from a large SUSY mass expansion of the one-particle irreducible functions in powers of $\left(p / M_{S U S Y}\right)^{n}$, with $p$ being any typical external momentum or mass of the order of the electroweak scale, and $M_{S U S Y}$ being the heavy SUSY masses $\left(M_{S U S Y} \gg m_{E W}\right)$. The leading contribution of zero order, $n=0$, corresponds to the exact result in the zero external momentum approximation. The advantage of using an effective action approach is that it provides not just the first term in the expansion but the whole series.

The main motivation of this work is to investigate with complete generality whether or not there is decoupling of heavy squarks and gluinos in the low energy effective interactions of the MSSM Higgs sector with the SM quarks. In particular we will concentrate on the effective Yukawa-like interactions $h^{o} b \bar{b}, h^{o} t \bar{t}, H^{o} b \bar{b}, H^{o} t \bar{t}, A^{o} b \bar{b}, A^{o} t \bar{t}$ and $H^{+} \bar{t} b$ that are generated by explicit integration of stops, sbottoms and gluinos to one-loop level and to order $\alpha_{S}$. We have focused on these SUSY-QCD third generation radiative corrections since they are the dominant ones [4-8].

The decoupling behavior of SUSY particles has been studied previously with complete generality for the effective electroweak gauge bosons self-interactions and within the 
same effective action approach in a series of works [9]. It has been shown, by explicit integration to one-loop level, that heavy squarks, sleptons, charginos and neutralinos do decouple in the low energy $W^{ \pm}, Z$ and $\gamma$ self-interactions. In agreement with the Appelquist-Carazzone theorem [10], this means that the heavy SUSY particles do not generate any new electroweak bosons interactions that were not already present at the SM Lagrangian, and all the terms found in the low energy expansion of the effective action indeed decouple as inverse powers of the heavy SUSY masses. The behavior of decoupling in the present context of Higgs-quark-quark interactions has been studied partially by several authors but none with complete generality. Some of these couplings were studied in the context of the zero external approximation in [11-14]. The radiatively generated momentum-dependent Yukawa couplings for the case of neutral Higgs bosons and light quarks with $m_{q} \ll m_{H}$, and for external on-shell particles, have been considered in [15]. In addition, there are several studies of the partial decay widths $\Gamma\left(h^{o} \rightarrow b \bar{b}\right)[16]$ and $\Gamma\left(H^{+} \rightarrow t \bar{b}\right)[17]$ in the limit of heavy SUSY particles, by using a Feynman diagrammatic approach. All these studies already indicate a non-decoupling behavior of the heavy SUSY particles in the Higgs sector of the MSSM. This is manifested as some non-vanishing contributions in the oneloop radiative corrections of the partial widths from genuine SUSY particles, which are present even in the case of infinitely heavy SUSY masses.

Here we study the decoupling behavior of SUSY particles in the effective field theory formalism. Indeed, we deduce the particular effective theory that remains at low energies after explicit integration in the path integral of the heavy SUSY modes in the MSSM theory. Our starting point with regard to the Higgs sector interactions with quarks is the well known tree-level MSSM Lagrangian, which belongs to the class of two Higgs doublet models of type II (2HDMII) [3] where one of the two doublets couples just to the top-like quarks and the other one just to the bottom-like quarks. At this level, the absence of other possible Yukawa couplings is indeed a consequence of the underlying supersymmetry. When integrating out SUSY particles, one could, in principle, find out different scenarios at low energies. One may end up either with effective interactions of the same type II or, in the most general case, one could generate new interactions belonging to the unrestricted type III models (2HDMIII) [18], where both doublets couple to both top and bottom-like quarks. It has been argued on general grounds $[16,17]$ that the most plausible scenario at low energies is a 2HDMIII, since the supersymmetry is broken by the heavy SUSY masses and, therefore, the restrictions imposed by this symmetry do not apply. However, the final answer can only be known once these effective low energy interactions have been explicitly computed. In case one gets a 2 HDMII one should conclude that there is indeed decoupling of the SUSY particles, since all their effects can be absorbed into redefinitions of the low energy parameters, couplings and fields. In contrast, if one gets a 2HDMIII one should conclude there is no decoupling of the heavy SUSY particles, since their effects cannot be eliminated by that redefinition. In this latter case, the new effective interactions do remain at low energies, even in the infinitely heavy SUSY masses limit and consequently, it may bring some hint of indirect SUSY signals.

The final goal of our work is precisely to compute, with complete generality, all these new effective Yukawa interactions in order to provide, first, a formal proof of SUSY nondecoupling behavior in Higgs physics and, second, the complete list with the particular values of the new $h^{o} b \bar{b}, h^{o} t \bar{t}, H^{o} b \bar{b}, H^{o} t \bar{t}, A^{o} b \bar{b}, A^{o} t \bar{t}$ and $H^{+} \bar{t} b$ Yukawa couplings, which in turn have interesting applications for phenomenology [11-17,19-23].

The paper is organized as follows. In Sec. II the Higgs and SUSY sectors together with the relevant interactions for the present work are briefly reviewed. In Sec. III we integrate out the squarks and gluino fields to one-loop level and $\mathcal{O}\left(\alpha_{s}\right)$ and we give the expressions for the non-local effective action. In Sec. IV we compute the effective local interactions of Higgs particles with quarks in the limit of heavy squarks and gluinos. Finally, Sec. V is devoted to a complete discussion of the results and to the conclusions.

\section{HIGGS, SUSY-QCD SECTORS AND RELEVANT INTERACTIONS IN THE MSSM}

In this section we briefly review the relevant MSSM spectrum and interactions for the present work which are the Higgs sector, the SUSY-QCD sector, the Yukawa Higgsquark-quark interactions, the squark-quark-gluino interactions and the Higgs-squark-squark interactions.

In the MSSM Higgs sector there are five physical Higgs particles: two $C P$-even neutral scalar particles $h^{o}, H^{o}$, one $C P$-odd neutral pseudoscalar particle $A^{\circ}$ and two charged scalar particles $H^{ \pm}$. Due to supersymmetry, the parameters of the Higgs sector are constrained and, at the tree level, they can be written in terms of just two MSSM parameters. These are commonly chosen to be the mass of the $C P$-odd neutral Higgs boson, $m_{A^{o}}$, and the ratio of the vacuum expectation values of the two Higgs doublets, $\tan \beta=v_{2} / v_{1}$. The Higgs bosons masses and the mixing angle in the neutral sector are given in terms of these two parameters, at the tree level, by

$$
\begin{aligned}
m_{H^{ \pm}}^{2}= & m_{A^{o}}^{2}+m_{W}^{2} \\
m_{H^{o}, h^{o}}^{2}= & \frac{1}{2}\left[m_{A^{o}}^{2}+m_{Z}^{2}\right. \\
& \left. \pm \sqrt{\left(m_{A^{o}}^{2}+m_{Z}^{2}\right)^{2}-4 m_{A^{o}}^{2} m_{Z}^{2} \cos ^{2} 2 \beta}\right] \\
\tan 2 \alpha= & \tan 2 \beta \frac{m_{A^{o}}^{2}+m_{Z}^{2}}{m_{A^{o}}^{2}-m_{Z}^{2}} .
\end{aligned}
$$

The case of large pseudoscalar mass, $m_{A}{ }^{o} \gg m_{E W}$, deserves special mention, because in this so-called decoupling limit [24] the $h^{o}$ boson resembles the SM Higgs boson.

Once the radiative corrections to the Higgs boson sector are included [25], the Higgs mass pattern changes slightly and depends on the other MSSM parameters as well. How- 
ever, irrespective of the particular values of the MSSM parameters, there is the important prediction of the lightest Higgs boson mass being below about $130 \mathrm{GeV}$, clearly within the reach of the present and future colliders.

The SUSY-QCD sector in the MSSM consists of squarks and gluinos. We focus here on the third-generation squarks, which will be the relevant ones for the dominant radiative corrections to the Yukawa couplings, and neglect possible intergenerational mixing. The tree-level stop and sbottom squared-mass matrices are

$$
\begin{aligned}
& \mathcal{M}_{\tilde{t}}^{2}=\left(\begin{array}{cc}
M_{L}^{2} & m_{t} X_{t} \\
m_{t} X_{t} & M_{R}^{2}
\end{array}\right), \\
& \mathcal{M}_{\tilde{b}}^{2}=\left(\begin{array}{cc}
M_{L}^{\prime 2} & m_{b} X_{b} \\
m_{b} X_{b} & M_{R}^{\prime 2}
\end{array}\right),
\end{aligned}
$$

where

$$
\begin{aligned}
M_{L}^{2} & =M_{\tilde{Q}}^{2}+m_{t}^{2}+\cos 2 \beta\left(1 / 2-2 / 3 s_{W}^{2}\right) m_{Z}^{2} \\
M_{R}^{2} & =M_{\widetilde{U}}^{2}+m_{t}^{2}+2 / 3 \cos 2 \beta s_{W}^{2} m_{Z}^{2} \\
X_{t} & =A_{t}-\mu \cot \beta \\
M_{L}^{\prime 2} & =M_{\tilde{Q}}^{2}+m_{b}^{2}-\cos 2 \beta\left(1 / 2-1 / 3 s_{W}^{2}\right) m_{Z}^{2} \\
M_{R}^{\prime 2} & =M_{\widetilde{D}}^{2}+m_{b}^{2}-1 / 3 \cos 2 \beta s_{W}^{2} m_{Z}^{2} \\
X_{b} & =A_{b}-\mu \tan \beta .
\end{aligned}
$$

Here $m_{t}$ and $m_{b}$ are the top and bottom quark masses, respectively, $m_{Z}$ and $m_{W}$ are the $Z$ and $W$ gauge boson masses, respectively, and $s_{W} \equiv \sin \theta_{W}$. The parameters $M_{\tilde{Q}}, M_{\tilde{D}}$ and $M_{\tilde{U}}$ are the soft-SUSY-breaking masses for the thirdgeneration $\mathrm{SU}(2)$ squark doublet $\left(\tilde{t}_{L}, \widetilde{b}_{L}\right)$ and the singlets $\widetilde{b}_{R}$ and $\tilde{t}_{R}$, respectively. $\tilde{q}_{L, R}$ are the superpartners of the chiral projections of quarks $q_{L, R}=P_{L, R} q$, respectively, where $P_{L, R}=\left(1 \mp \gamma_{5}\right) / 2 . \quad A_{b, t}$ are the corresponding soft-SUSYbreaking trilinear couplings and $\mu$ is the bilinear coupling of the two Higgs doublets. The squarks mass eigenstates are given by

$$
\left(\begin{array}{c}
\tilde{q}_{1} \\
\tilde{q}_{2}
\end{array}\right)=\left(R_{q}\right)^{-1}\left(\begin{array}{c}
\tilde{q}_{L} \\
\tilde{q}_{R}
\end{array}\right),
$$

where

$$
R_{q}=\left(\begin{array}{cc}
c_{q} & -s_{q} \\
s_{q} & c_{q}
\end{array}\right)
$$

and $c_{q}=\cos \theta_{q}, s_{q}=\sin \theta_{q}$ with $q=t, b$. The stop and sbottom mass eigenvalues are given by

$$
M_{\tilde{t}_{1,2}}^{2}=\frac{1}{2}\left[M_{L}^{2}+M_{R}^{2} \pm \sqrt{\left(M_{L}^{2}-M_{R}^{2}\right)^{2}+4 m_{t}^{2} X_{t}^{2}}\right],
$$

$$
M_{\tilde{b}_{1,2}}^{2}=\frac{1}{2}\left[M_{L}^{\prime 2}+M_{R}^{\prime 2} \pm \sqrt{\left(M_{L}^{\prime 2}-M_{R}^{\prime 2}\right)^{2}+4 m_{b}^{2} X_{b}^{2}}\right] .
$$

And the squark mixing angles $\theta_{q}(q=t, b)$ are given by

$$
\begin{aligned}
& \cos 2 \theta_{t}=\frac{M_{L}^{2}-M_{R}^{2}}{M_{\tilde{t}_{1}}^{2}-M_{\tilde{t}_{2}}^{2}}, \quad \cos 2 \theta_{b}=\frac{M_{L}^{\prime 2}-M_{R}^{\prime 2}}{M_{\tilde{b}_{1}}^{2}-M_{\tilde{b}_{2}}^{2}}, \\
& \sin 2 \theta_{q}=\frac{2 m_{q} X_{q}}{M_{\tilde{q}_{1}}^{2}-M_{\tilde{q}_{2}}^{2}} .
\end{aligned}
$$

The free Lagrangian for Higgs bosons, quarks, squarks and Majorana gluinos in the physical basis are given, respectively, by

$$
\begin{aligned}
\mathcal{L}_{0}(H)= & \frac{1}{2} \sum_{H}\left(\partial_{\mu} H \partial^{\mu} H-H m_{H}^{2} H\right) \\
\mathcal{L}_{0}(q)= & \bar{b}\left(i \not b-m_{b}\right) b+\bar{t}\left(i b-m_{t}\right) t \\
\mathcal{L}_{0}(\tilde{q})= & \sum_{i=1,2}\left(\partial_{\mu} \widetilde{b}_{i}^{*} \partial^{\mu} \widetilde{b}_{i}-M_{\widetilde{b}_{i}}^{2} \widetilde{b}_{i}^{*} \widetilde{b}_{i}\right) \\
& +\sum_{i=1,2}\left(\partial_{\mu} \widetilde{t}_{i}^{*} \partial^{\mu} \widetilde{t}_{i}-M_{\tilde{t}_{i}}^{2} \widetilde{t}_{i}^{*} \widetilde{t}_{i}\right) \\
\mathcal{L}_{0}(\tilde{g})= & \frac{1}{2} \overline{\tilde{g}}\left(i \not b-M_{\tilde{g}}\right) \tilde{g},
\end{aligned}
$$

where the sum in $H$ runs along the Higgs fields $H$ $=h^{o}, H^{o}, A^{o}, H^{1}, H^{2}$, and $H^{1}, H^{2}$ are related to the physical charged Higgs bosons by $H^{1}=\left(H^{+}+H^{-}\right) / \sqrt{2}, H^{2}=$ $-i\left(H^{+}-H^{-}\right) / \sqrt{2}$ and $m_{H^{1}}^{2}=m_{H^{2}}^{2}=m_{H^{ \pm}}^{2} . M_{\tilde{g}}$ is the gluino mass, and we have omitted for brevity the color indices in the gluinos, quarks and squarks fields. Notice that in order to make more explicit our posterior assumption on the mass spectrum, we have denoted by little $m$ all the light masses of the order of $m_{E W}$, and by capital $M$ all the heavy masses of the order of $M_{S U S Y}$.

The tree-level Yukawa Higgs-quark-quark interactions in the MSSM are like in a general 2HDMII:

$$
\begin{aligned}
\mathcal{L}(H, q)= & \frac{g m_{b} \sin \alpha}{2 m_{W} \cos \beta} h^{o} \bar{b} b-\frac{g m_{t} \cos \alpha}{2 m_{W} \sin \beta} h^{o} \bar{t} t \\
& -\frac{g m_{b} \cos \alpha}{2 m_{W} \cos \beta} H^{o} \bar{b} b-\frac{g m_{t} \sin \alpha}{2 m_{W} \sin \beta} H^{o} \bar{t} t \\
& +\frac{i g m_{b}}{2 m_{W}} \tan \beta A^{o} \bar{b} \gamma_{5} b+\frac{i g m_{t}}{2 m_{W}} \cot \beta A^{o} \bar{t} \gamma_{5} t \\
& +\frac{g m_{b}}{\sqrt{2} m_{W}} \tan \beta\left(H^{+} \bar{t}_{L} b_{R}+\text { H.c. }\right) \\
& +\frac{g m_{t}}{\sqrt{2} m_{W}} \cot \beta\left(H^{+} \bar{t}_{R} b_{L}+\text { H.c. }\right) .
\end{aligned}
$$


The MSSM gluino-squark-quark interactions terms, in the chiral basis, are

$$
\begin{aligned}
\mathcal{L}(\tilde{g}, \tilde{q}, q)= & -\sqrt{2} g_{s} t\left(\overline{\tilde{g}} \widetilde{t}_{L}^{*} t_{L}+\overline{\tilde{g}} \widetilde{b}_{L}^{*} b_{L}\right. \\
& \left.-\overline{\tilde{g}} \widetilde{t}_{R}^{*} t_{R}-\overline{\tilde{g}} \widetilde{b}_{R}^{*} b_{R}\right)+ \text { H.c. }
\end{aligned}
$$

where $t \overline{\tilde{g}} \tilde{q}^{*} q \equiv t_{i j}^{a} \overline{\tilde{g}}_{a} \tilde{q}^{* i} q^{j}$, and $t_{i j}^{a}$ are the standard $S U(3)_{c}$ generators. We will omit the color indices from now on.

Finally, the Lagrangian terms involving Higgs-squarksquark interactions, in the chiral squarks basis, are

$$
\begin{aligned}
\mathcal{L}(H, \tilde{q})= & \sum_{i, j, H_{n}}\left[\left(a_{H_{n}}^{\tilde{t}}\right)_{i j} H_{n} \tilde{t}_{i}^{*} \tilde{t}_{j}+\left(a_{H_{n}}^{\tilde{b}}\right)_{i j} H_{n} \widetilde{b}_{i}^{*} \widetilde{b}_{j}\right] \\
& +\sum_{i, j, H_{c}}\left[\left(a_{H_{c}}\right)_{i j} H_{c} \widetilde{t}_{i}^{*} \widetilde{b}_{j}+\text { H.c. }\right]
\end{aligned}
$$

where the $i$ and $j$ indices run along $L, R$; the Higgs fields $H_{n}$ along $H_{n}=h^{o}, H^{o}, A^{o}$ and $H_{c}$ along $H_{c}=H^{1}, H^{2}$. The expressions for the couplings $a_{H}$ are given in Appendix A.

\section{INTEGRATION OF SQUARKS AND GLUINOS}

In this section we perform the integration to one-loop level and to $\mathcal{O}\left(\alpha_{s}\right)$ of the squarks and gluinos in the MSSM by using the standard functional techniques. Since we are studying here the effect of the SUSY-QCD sector on the Yukawa Higgs-quark-quark interactions, we take just the relevant terms in the MSSM action, which can be generically written as

$$
\begin{aligned}
S[H, q, \tilde{q}, \tilde{g}]= & S_{0}[H]+S_{0}[q]+S_{0}[\tilde{q}]+S_{0}[\tilde{g}]+S[H, q] \\
& +S[\tilde{g}, \tilde{q}, q]+S[H, \tilde{q}],
\end{aligned}
$$

where the $S_{0}$ and $S$ are given in terms of the free and interaction Lagrangians, respectively, of Eqs. (8), (9), (10) and (11) by

$$
\begin{aligned}
S_{0}[\Phi] & =\int d x \mathcal{L}_{0}(\Phi), \quad \Phi=H, q, \tilde{q}, \tilde{g}, \\
S[H, q] & =\int d x \mathcal{L}(H, q), \\
S[\tilde{g}, \tilde{q}, q] & =\int d x \mathcal{L}(\tilde{g}, \tilde{q}, q), \\
S[H, \tilde{q}] & =\int d x \mathcal{L}(H, \tilde{q}),
\end{aligned}
$$

and $d x$ is the ordinary space-time integration measure.

In the following we introduce some convenient compact notation for the functional integration. The chiral quark fields, their squarks partners and the physical squarks are grouped here as

$$
Q=\left(\begin{array}{c}
t_{L} \\
t_{R} \\
b_{L} \\
b_{R}
\end{array}\right), \quad \widetilde{Q}_{c h}=\left(\begin{array}{c}
\tilde{t}_{L} \\
\tilde{t}_{R} \\
\widetilde{b}_{L} \\
\tilde{b}_{R}
\end{array}\right), \quad \widetilde{Q}=\left(\begin{array}{c}
\tilde{t}_{1} \\
\tilde{t}_{2} \\
\tilde{b}_{1} \\
\tilde{b}_{2}
\end{array}\right) .
$$

The two squark basis are related via an orthogonal $4 \times 4$ matrix $R$, given in terms of the matrices $R_{q}(q=t, b)$ of Eq. (5), by

$$
\widetilde{Q}_{c h}=R \widetilde{Q}, \quad R=\left(\begin{array}{cc}
R_{t} & 0 \\
0 & R_{b}
\end{array}\right)
$$

With regard to the gluinos, which are Majorana spinors, we will use a Majorana representation for the $\gamma$ Dirac matrices so that the Majorana fields will be real, $\gamma_{i}(i=1,2,3)$ imaginary and symmetric, $\gamma_{0}$ and $\gamma_{5}$ imaginary and antisymmetric and the chiral projectors Hermitian.

With this notation, the squark and gluino dependent terms of the above action can be written in terms of the physical states as follows. The free squark action is

$$
S_{0}[\tilde{q}]=\int d x \widetilde{Q}^{\dagger}\left(-\square-M_{\tilde{q}}^{2}\right) \widetilde{Q} \equiv\left\langle\widetilde{Q}^{\dagger} A_{\tilde{q}}^{0} \widetilde{Q}\right\rangle,
$$

where $M_{\tilde{q}}^{2} \equiv \operatorname{diag}\left(M_{\tilde{t}_{1}}^{2}, M_{\tilde{t}_{2}}^{2}, M_{\tilde{b}_{1}}^{2}, M_{\tilde{b}_{2}}^{2}\right)$ and $\square \equiv \partial_{\mu} \partial^{\mu}$. The free action for the Majorana gluino fields is

$$
S_{0}[\tilde{g}]=\int d x \frac{1}{2} \overline{\tilde{g}}\left(i \not b-M_{\tilde{g}}\right) \tilde{g} \equiv \frac{1}{2}\left\langle\overline{\tilde{g}} A_{\tilde{g}}^{0} \tilde{g}\right\rangle .
$$

The gluino-squark-quark interactions are written in compact notation as

$$
\begin{aligned}
S[\tilde{g}, \tilde{q}, q] & =\int d x\left(-\sqrt{2} g_{s} t \overline{\tilde{g}} \widetilde{Q}^{\dagger} R^{T} \Sigma Q+\text { H.c. }\right) \\
& \equiv\left\langle\overline{\tilde{g}} \widetilde{Q}^{\dagger} O Q\right\rangle+\text { H.c., }
\end{aligned}
$$

where we have introduced the matrix $\Sigma \equiv \operatorname{diag}(1,-1,1,-1)$ and the interaction operator is defined as

$$
O \equiv-\sqrt{2} g_{s} t R^{T} \Sigma .
$$

The Higgs-squark-squark interactions are given by ${ }^{1}$

$$
S[H, \tilde{q}]=\sum_{H} \int d x H \widetilde{Q}^{\dagger} B_{H} \widetilde{Q} \equiv\left\langle H \widetilde{Q}^{\dagger} B_{H} \widetilde{Q}\right\rangle,
$$

\footnotetext{
${ }^{1}$ Since we are not going to integrate here the Higgs fields, we work in the unitary gauge and refer always to the physical Higgs basis.
} 
where $H$ runs again along $H=h^{o}, H^{o}, A^{o}, H^{1}, H^{2}, B_{H}$ $\equiv R^{T} A_{H} R$ and $A_{H}$ are the $4 \times 4$ coupling matrices that are defined in Appendix A.

We next outline the computation of the contribution to the quarks and Higgs bosons effective action coming from the functional integration of the squark and gluino fields, $\Delta \Gamma_{e f f}[H, q]$. This integration can be written as

$$
\begin{aligned}
e^{i \Delta \Gamma_{e f f}[H, q]}= & \int\left[d \widetilde{Q}^{\dagger}\right][d \widetilde{Q}][d \tilde{g}] \\
& \times e^{i\left(S_{0}[\tilde{q}]+S_{0}[\tilde{g}]+S[\tilde{g}, \tilde{q}, q]+S[H, \tilde{q}]\right)} .
\end{aligned}
$$

After this integration the total effective action for quarks and Higgs particles will then be obtained by

$$
\Gamma_{e f f}[H, q]=S_{0}[H]+S_{0}[q]+S[H, q]+\Delta \Gamma_{e f f}[H, q] .
$$

In order to perform first the gluino integration we write Eq. (21) as

$$
\begin{aligned}
e^{i \Delta \Gamma_{e f f}[H, q]}= & \int\left[d \widetilde{Q}^{\dagger}\right][d \widetilde{Q}] e^{i\left\langle\widetilde{Q}^{\dagger}\left(A_{\tilde{q}}^{0}+H B_{H}\right) \widetilde{Q}\right\rangle} \\
& \times \int[d \tilde{g}] e^{i(1 / 2)\left\langle\overline{\tilde{g}} A_{\tilde{g}}^{0} g\right\rangle+i\left(\left\langle\overline{\tilde{g}} \widetilde{Q}^{\dagger} O Q\right\rangle+\text { H.c. }\right)} .
\end{aligned}
$$

A standard Gaussian integration of the real Grassmann variable $\tilde{g}$ yields

$$
\begin{aligned}
e^{i \Delta \Gamma_{e f f}[H, q]}= & \int\left[d \widetilde{Q}^{\dagger}\right][d \widetilde{Q}] e^{i\left\langle\widetilde{Q}^{\dagger}\left(A_{\tilde{q}}^{0}+H B_{H}\right) \widetilde{Q}\right\rangle} \\
& \times e^{-(i / 2)\left\langle\bar{\eta}\left(A_{\tilde{g}}^{0}\right)^{-1} \eta\right\rangle},
\end{aligned}
$$

where we have omitted a field independent global factor that is irrelevant for the effective action, and we have introduced the Majorana field

$$
\eta \equiv \widetilde{Q}^{\dagger} O Q+Q^{\dagger} O^{\dagger} \widetilde{Q}
$$

Thus, we have

$$
\begin{aligned}
e^{i \Delta \Gamma_{e f f}[H, q]}= & \int\left[d \widetilde{Q}^{\dagger}\right][d \widetilde{Q}] \\
& \times e^{i\left\langle\widetilde{Q}^{\dagger}\left(A_{\tilde{q}}^{0}+H B_{H}-O Q \gamma_{0} D_{g}^{\sim} Q^{\dagger} O^{\dagger}\right) \widetilde{Q}\right\rangle} \\
= & {\left[\operatorname{det}\left(A_{\tilde{q}}^{0}+H B_{H}-O Q \gamma_{0} D_{\tilde{g}} Q^{\dagger} O^{\dagger}\right)\right]^{-1}, }
\end{aligned}
$$

where we have performed another complex-scalar Gaussian integration and $D_{\tilde{g}}=\left(A_{\tilde{g}}^{0}\right)^{-1}$ is the gluino propagator which, in the position representation, can be written as

$$
D_{\tilde{g} x y}=\int d \tilde{k} \frac{e^{-i k(x-y)}}{k-M_{\tilde{g}}},
$$

where $d \tilde{k} \equiv d^{4} k /(2 \pi)^{4}$. Therefore, aside from a field independent term, the contribution to the effective action can be written as

$$
\Delta \Gamma_{e f f}[H, q]=i \operatorname{Tr} \log \left[1+D_{\tilde{q}}^{\sim}\left(H B_{H}-O Q \gamma_{0} D_{g}^{\sim} Q^{\dagger} O^{\dagger}\right)\right],
$$

where $\operatorname{Tr}$ is the functional trace, and the squark propagator $D_{\tilde{q}}=\left(A_{\tilde{q}}^{0}\right)^{-1}$ is given, in the position representation, by

$$
D_{\tilde{q} x y}=\int d \tilde{q} \frac{e^{-i q(x-y)}}{q^{2}-M_{\tilde{q}}^{2}}
$$

where

$$
\frac{1}{q^{2}-M_{\tilde{q}}^{2}} \equiv \operatorname{diag}\left(\frac{1}{q^{2}-M_{\tilde{t}_{1}}^{2}}, \frac{1}{q^{2}-M_{\tilde{t}_{2}}^{2}}, \frac{1}{q^{2}-M_{\tilde{b}_{1}}^{2}}, \frac{1}{q^{2}-M_{\tilde{b}_{2}}^{2}}\right)
$$

Expanding the logarithm in Eq. (28) we get

$$
\begin{aligned}
\Delta \Gamma_{e f f}[H, q]= & i \sum_{k=1}^{\infty} \frac{(-)^{k+1}}{k} \\
& \times \operatorname{Tr}\left[D_{g}^{\sim}\left(H B_{H}-O Q \gamma_{0} D_{\tilde{g}}^{\sim} Q^{\dagger} O^{\dagger}\right)\right]^{k} \\
\equiv & \sum_{k=1}^{\infty} \Delta \Gamma_{\text {eff }}^{(k)} .
\end{aligned}
$$

In this work we are interested in the possible one-loop effects of the SUSY-QCD sector on the Higgs couplings to quark pairs and, therefore, only a few terms of this expansion will be taken into account. For $k=1$ we have

$$
\Delta \Gamma_{e f f}^{(1)}=i \operatorname{Tr} D_{\tilde{q}}^{\sim}\left(H B_{H}-O Q \gamma_{0} D_{\tilde{g}} Q^{\dagger} O^{\dagger}\right),
$$

which in the position representation reads

$$
\begin{aligned}
\Delta \Gamma_{e f f}^{(1)}= & i \operatorname{tr} \sum_{H} \int d x D_{\tilde{q} x x}\left(H_{x} B_{H}\right) \\
& -i \operatorname{tr} \int d x d y D_{\tilde{q} x y} O Q_{y} \gamma_{0} D_{\tilde{g} y x} Q_{x}^{\dagger} O^{\dagger},
\end{aligned}
$$

where the notation for the fields is $H_{x}=H(x), Q_{y}=Q(y)$, etc. The first term corresponds to the Higgs tadpoles with squark loops which are of zero order in $\alpha_{s}$ and, hence, will not be considered in the following. The second term gives the one-loop contributions to the quark propagators which are of order $\alpha_{s}$ and involves both a gluino and a squark propagator in the loop. By substituting the previous expres- 
sions for the operator $O$ and for the squark and gluino propagators, and after some algebra, this second term can be written as

$$
\Delta \Gamma_{e f f}^{(1)}=\frac{2 \alpha_{s}}{3 \pi} \int d x d y \sum_{a b c} \bar{Q}_{x}^{c}(\Sigma R)_{c a} \Pi_{x y}^{a}\left(R^{T} \Sigma\right)_{a b} Q_{y}^{b},
$$

where

$$
\Pi_{x y}^{a} \equiv \int d \tilde{p} e^{-i p(x-y)}\left[p B_{1}+M_{\tilde{g}} B_{0}\right]\left(p^{2}, M_{\tilde{g}}^{2}, M_{\tilde{q}_{a}}^{2}\right)
$$

and the indices $a, b, c$ run along the four entries corresponding to the quarks and the (physical) squarks matrices in Eq. (15).

The $k=2$ contribution is given by

$$
\begin{aligned}
\Delta \Gamma_{e f f}^{(2)} & =-\frac{i}{2} \operatorname{Tr}\left[D_{\tilde{q}}\left(H B_{H}-O Q \gamma_{0} D_{\tilde{g}} Q^{\dagger} O^{\dagger}\right)\right]^{2} \\
& =i \operatorname{Tr}\left(H B_{H} D_{\tilde{q}} O Q \gamma_{0} D_{\tilde{g}} Q^{\dagger} O^{\dagger} D_{\tilde{q}}\right)
\end{aligned}
$$

where in the last equality we have omitted those terms that do not contribute to the Higgs-quark-quark couplings. In the position representation this last term can be written as

$$
\begin{aligned}
\Delta \Gamma_{e f f}^{(2)}= & i \operatorname{tr} \sum_{H} \int d x d y d z \\
& \times H_{x} B_{H} D_{\tilde{q} x y} O Q_{y} \gamma_{0} D_{\tilde{g} y z} Q_{z}^{\dagger} O^{\dagger} D_{\tilde{q} z x} .
\end{aligned}
$$

Finally, by substituting the operator $O$ and the squark and gluino propagators of Eqs. (19), (27) and (29) in Eq. (36), and after some algebra, this contribution to the effective action can be written in terms of the standard one-loop integrals as

$$
\begin{aligned}
\Delta \Gamma_{e f f}^{(2)}= & -\frac{2 \alpha_{s}}{3 \pi} \sum_{H} \int d x d y d z \\
& \times \sum_{a b c d} \bar{Q}_{z}^{d}(\Sigma R)_{d a} H_{x} B_{H}^{a b} V_{x y z}^{a b}\left(R^{T} \Sigma\right)_{b c} Q_{y}^{c},
\end{aligned}
$$

where

$$
\begin{aligned}
V_{x y z}^{a b} \equiv & \int d \tilde{q} d \tilde{p} e^{-i q(x-y)} e^{-i p(z-y)} \\
& \times\left[\not p C_{11}+\not C_{12}+M_{\tilde{g}} C_{0}\right] \\
& \times\left(p^{2}, q^{2}, r^{2}, M_{\tilde{g}}^{2}, M_{\tilde{q}_{a}}^{2}, M_{\tilde{q}_{b}}^{2}\right)
\end{aligned}
$$

with $r \equiv-(p+q)$. Here again, the indices $a, b, c, d$ run along the four entries of the quark and squark matrices and $H$ runs along the Higgs fields $H=h^{o}, H^{o}, A^{o}, H^{1}, H^{2}$. The last parenthesis refers to the arguments of the scalar three-point inte-
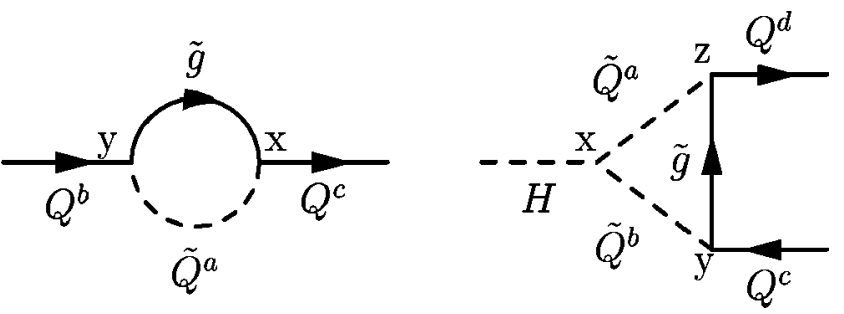

FIG. 1. Diagrams corresponding to $\Delta \Gamma_{e f f}^{(1)}$ and $\Delta \Gamma_{e f f}^{(2)}$ of Eqs. (33), (34) and (37), (38), respectively.

grals. Our definition of the scalar one-loop integrals $B_{0}, B_{1}, C_{0}, C_{11}$ and $C_{12}$ is as in [26] and is given for completeness in Appendix B.

The corresponding generic Feynman diagrams for $\Delta \Gamma_{e f f}^{(1)}$ and $\Delta \Gamma_{\text {eff }}^{(2)}$ of Eqs. (33), (34) and (37), (38), respectively, are shown in Fig. 1.

\section{EFFECTIVE HIGGS-QUARK-QUARK INTERACTIONS FROM HEAVY SQUARKS AND GLUINOS}

In this section we compute the effective local interactions of Higgs particles with quarks to one-loop level and $\mathcal{O}\left(\alpha_{s}\right)$, in the limit of heavy squarks and gluinos. We derive the quarks and Higgs bosons effective Lagrangian starting from the nonlocal effective action of Eqs. (22), (30), (33), (34), (37), and (38) which includes the contributions coming from integrating out the squarks and gluino fields. Next we perform a large $M_{S U S Y}$ expansion that is valid in the limit of nearly degenerate and very heavy SUSY particles, $M_{\tilde{g}} \sim M_{\tilde{q}}$ $\sim M_{S U S Y} \gg m_{E W}$, where $m_{E W}$ stands for any mass or external momentum associated with a non-SUSY particle, i.e. quarks, gauge bosons and Higgs particles. For that purpose, we consider all the SUSY mass parameters and the $\mu$ parameter of the same order (collectively denoted by $M_{S U S Y}$ ) and much heavier than the electroweak scale, ${ }^{2}$ that is, $M_{S U S Y} \sim M_{\tilde{g}}$ $\sim M_{\tilde{Q}} \sim M_{\tilde{U}} \sim M_{\tilde{D}} \sim \mu \gg m_{E W}$.

The effective Lagrangian is then defined in the usual way,

$$
\Gamma_{e f f}[H, q] \equiv \int d x \mathcal{L}_{e f f}(H, q),
$$

and, correspondingly to Eq. (22), it can be written as follows:

$$
\begin{aligned}
\mathcal{L}_{\text {eff }}(H, q)= & \mathcal{L}_{0}(H)+\mathcal{L}_{0}(q)+\mathcal{L}(H, q) \\
& +\Delta \mathcal{L}_{\text {eff }}(H, q) .
\end{aligned}
$$

In order to compute $\Delta \mathcal{L}_{e f f}(H, q)$ to one-loop order, $\mathcal{O}\left(\alpha_{s}\right)$, and in the limit of heavy squarks and gluinos, we substitute into Eqs. (33), (34), (37) and (38) the correspond-

\footnotetext{
${ }^{2}$ Notice that our choice of large $\mu$ is not necessary for a heavy SUSY-QCD spectrum but it will be mandatory to get all the gauginos heavy in the SUSY-electroweak sector. Although it is not needed for the present work, we are thinking of the whole SUSY spectrum being heavy as compared to the electroweak scale.
} 
ing interaction matrices $\Sigma, R$ and $B_{H}$ and the expressions for the large mass expansion of the one-loop integrals and mixing angles given in Appendix B. After some algebra, we obtain the following result:

$$
\begin{aligned}
& \Delta \mathcal{L}_{e f f}(H, q)=\frac{\alpha_{s}}{3 \pi} \Delta \bar{b}(i \not b) b+\frac{\alpha_{s}}{3 \pi} \frac{M_{\tilde{g}}\left(A_{b}-\mu \tan \beta\right)}{M_{S U S Y}^{2}} m_{b} \bar{b} b+\frac{\alpha_{s}}{3 \pi} \Delta \bar{t}(i \not b) t+\frac{\alpha_{s}}{3 \pi} \frac{M_{\tilde{g}}\left(A_{t}-\mu \cot \beta\right)}{M_{S U S Y}^{2}} m_{t} \bar{t} t \\
& -\frac{\alpha_{s}}{3 \pi} \frac{g m_{b} \sin \alpha}{2 m_{W} \cos \beta} \frac{M_{\tilde{g}}\left(A_{b}+\mu \cot \alpha\right)}{M_{S U S Y}^{2}} h^{o} \bar{b} b+\frac{\alpha_{s}}{3 \pi} \frac{g m_{t} \cos \alpha}{2 m_{W} \sin \beta} \frac{M_{\tilde{g}}\left(A_{t}+\mu \tan \alpha\right)}{M_{S U S Y}^{2}} h^{o} \bar{t} t \\
& +\frac{\alpha_{s}}{3 \pi} \frac{g m_{b} \cos \alpha}{2 m_{W} \cos \beta} \frac{M_{\tilde{g}}\left(A_{b}-\mu \tan \alpha\right)}{M_{S U S Y}^{2}} H^{o} \bar{b} b+\frac{\alpha_{s}}{3 \pi} \frac{g m_{t} \sin \alpha}{2 m_{W} \sin \beta} \frac{M_{\tilde{g}}\left(A_{t}-\mu \cot \alpha\right)}{M_{S U S Y}^{2}} H^{o} \bar{t} t \\
& -\frac{\alpha_{s}}{3 \pi} \frac{i g m_{b}}{2 m_{W}} \tan \beta \frac{M_{\tilde{g}}\left(A_{b}+\mu \cot \beta\right)}{M_{S U S Y}^{2}} A^{o} \bar{b} \gamma_{5} b-\frac{\alpha_{s}}{3 \pi} \frac{i g m_{t}}{2 m_{W}} \cot \beta \frac{M_{\tilde{g}}\left(A_{t}+\mu \tan \beta\right)}{M_{S U S Y}^{2}} A^{o} \bar{t} \gamma_{5} t \\
& -\frac{\alpha_{s}}{3 \pi} \frac{g m_{b}}{\sqrt{2} m_{W}} \tan \beta \frac{M_{\tilde{g}}\left(A_{b}+\mu \cot \beta\right)}{M_{S U S Y}^{2}}\left(H^{+} \bar{t}_{L} b_{R}+\text { H.c. }\right)-\frac{\alpha_{s}}{3 \pi} \frac{g m_{t}}{\sqrt{2} m_{W}} \cot \beta \frac{M_{\tilde{g}}\left(A_{t}+\mu \tan \beta\right)}{M_{S U S Y}^{2}} \\
& \times\left(H^{+} \bar{t}_{R} b_{L}+\text { H.c. }\right)+\mathcal{O}\left(\frac{m_{E W}}{M_{S U S Y}}\right)^{n}
\end{aligned}
$$

Some comments are in order. First notice that, as expected, the SUSY-QCD effects summarized in Eq. (41) include both the divergent and the finite contributions from heavy squarks and gluinos. We have used dimensional regularization to perform the one-loop integrals with $\Delta \equiv 2 / \epsilon$ $-\gamma_{E}+\log (4 \pi)-\log \left(M_{S U S Y}^{2} / \mu_{0}^{2}\right), \epsilon \equiv 4-D$ and $\mu_{0}$ is the usual mass scale of dimensional regularization. ${ }^{3}$ Second, all these leading terms are of $\mathcal{O}\left(m_{E W} / M_{S U S Y}\right)^{0}$, apart from the logarithms, in the large $M_{S U S Y}$ expansion. The last contribution in Eq. (41), not shown explicitly, refers to the next to leading order terms in the large $M_{S U S Y}$ expansion which are suppressed by inverse powers of $M_{S U S Y}(n>0)$ and vanish in the asymptotic $M_{S U S Y} \rightarrow \infty$ limit. It is worth mentioning here that, with this effective action formalism, one can easily get as well these next to leading order contributions by simply including the next terms in the expansions of the one-loop integrals and mixing angles. We will not work out here these terms but concentrate just on the leading terms which are the only ones that remain in the asymptotic large $M_{S U S Y}$ limit. For a discussion on the next to leading order terms we refer the reader to $[16,17]$ where they have been obtained for the $h^{\circ} \bar{b} b$ and $H^{+} \bar{t} b$ couplings, using a Feynman diagrammatic approach.

In order to study the decoupling behavior of the SUSYQCD sector in the Yukawa Higgs-quark-quark couplings we

\footnotetext{
${ }^{3}$ Notice that, presumably, the same results would have been obtained by using instead dimensional reduction.
}

have to find out if the effects found in Eq. (41) can be absorbed or not into redefinitions of the tree-level masses, couplings and wave functions of the light fields, namely, $H$ and $q$. We first redefine the quark fields and masses such that their corresponding kinetic and mass terms get the canonical form

$$
\begin{aligned}
b & =\left(1-\frac{\alpha_{s}}{6 \pi} \Delta\right) b^{\prime} \\
t & =\left(1-\frac{\alpha_{s}}{6 \pi} \Delta\right) t^{\prime} \\
m_{b} & =\left(1+\frac{\alpha_{s}}{3 \pi} \Delta+\frac{\alpha_{s}}{3 \pi} \frac{M_{\tilde{g}}\left(A_{b}-\mu \tan \beta\right)}{M_{S U S Y}^{2}}\right) m_{b}^{\prime} \\
m_{t} & =\left(1+\frac{\alpha_{s}}{3 \pi} \Delta+\frac{\alpha_{s}}{3 \pi} \frac{M_{g}\left(A_{t}-\mu \cot \beta\right)}{M_{S U S Y}^{2}}\right) m_{t}^{\prime} .
\end{aligned}
$$

After this, the remaining contributions in $\Delta \mathcal{L}_{\text {eff }}$ are rewritten in terms of these new fields $q^{\prime}$ and masses $m_{q}^{\prime}$, and we finally get 


$$
\begin{aligned}
\mathcal{L}_{e f f}\left(H, q^{\prime}\right)= & \mathcal{L}_{0}(H)+\bar{b}^{\prime}\left(i b-m_{b}^{\prime}\right) b^{\prime}+\bar{t}^{\prime}\left(i b-m_{t}^{\prime}\right) t^{\prime}+\frac{g m_{b}^{\prime} \sin \alpha}{2 m_{W} \cos \beta}\left[1-\frac{\alpha_{s}}{3 \pi} \frac{M_{\tilde{g}} \mu}{M_{S U S Y}^{2}}(\tan \beta+\cot \alpha)\right] h^{o} \bar{b}^{\prime} b^{\prime}-\frac{g m_{t}^{\prime} \cos \alpha}{2 m_{W} \sin \beta} \\
& \times\left[1-\frac{\alpha_{s}}{3 \pi} \frac{M_{\tilde{g}} \mu}{M_{S U S Y}^{2}}(\cot \beta+\tan \alpha)\right] h^{o} \bar{t}^{\prime} t^{\prime}-\frac{g m_{b}^{\prime} \cos \alpha}{2 m_{W} \cos \beta}\left[1-\frac{\alpha_{s}}{3 \pi} \frac{M_{\tilde{g}} \mu}{M_{S U S Y}^{2}}(\tan \beta-\tan \alpha)\right] H^{o} \bar{b}^{\prime} b^{\prime}-\frac{g m_{t}^{\prime} \sin \alpha}{2 m_{W} \sin \beta} \\
& \times\left[1-\frac{\alpha_{s}}{3 \pi} \frac{M_{\tilde{g}} \mu}{M_{S U S Y}^{2}}(\cot \beta-\cot \alpha)\right] H^{o} \bar{t}^{\prime} t^{\prime}+\frac{i g m_{b}^{\prime}}{2 m_{W}} \tan \beta\left[1-\frac{\alpha_{s}}{3 \pi} \frac{M_{\tilde{g}} \mu}{M_{S U S Y}^{2}}(\tan \beta+\cot \beta)\right] A^{o} \bar{b}^{\prime} \gamma_{5} b^{\prime} \\
& +\frac{i g m_{t}^{\prime}}{2 m_{W}} \cot \beta\left[1-\frac{\alpha_{s}}{3 \pi} \frac{M_{\tilde{g}} \mu}{M_{S U S Y}^{2}}(\tan \beta+\cot \beta)\right] A^{o} \bar{t}^{\prime} \gamma_{5} t^{\prime}+\frac{g m_{b}^{\prime}}{\sqrt{2} m_{W}} \tan \beta\left[1-\frac{\alpha_{s}}{3 \pi} \frac{M_{\tilde{g}} \mu}{M_{S U S Y}^{2}}(\tan \beta+\cot \beta)\right] \\
& \times\left(H^{+} \bar{t}_{L}^{\prime} b_{R}^{\prime}+\text { H.c. }\right)+\frac{g m_{t}^{\prime}}{\sqrt{2} m_{W}} \cot \beta\left[1-\frac{\alpha_{s}}{3 \pi} \frac{M_{\tilde{g}} \mu}{M_{S U S Y}^{2}}(\tan \beta+\cot \beta)\right]\left(H^{+} \bar{t}_{R}^{\prime} b_{L}^{\prime}+\text { H.c. }\right) .
\end{aligned}
$$

Notice that, as expected, the Higgs fields and Higgs masses do not get corrections from the SUSY-QCD sector at the one-loop level and $\mathcal{O}\left(\alpha_{s}\right)$, while the quark fields and quark masses do get corrections from this sector. Notice also that the final result is independent of the trilinear soft-breaking parameters, $A_{t, b}$.

The most relevant result is that we have found new Yukawa interactions which were not present in the tree-level interaction Lagrangian, $\mathcal{L}(H, q)$, and that cannot be absorbed into redefinitions of the masses, couplings and wave functions of the light fields. In order to illustrate this more clearly, we rewrite $\mathcal{L}_{\text {eff }}$ in terms of a set of new Yukawa couplings, $\Delta \lambda$,

$$
\begin{aligned}
\mathcal{L}_{e f f}(H, q)= & \mathcal{L}_{0}(H)+\mathcal{L}_{0}(q)+\mathcal{L}(H, q) \\
& +\Delta \lambda^{h^{o} \bar{b} b} h^{o} \bar{b} b+\Delta \lambda^{h^{o} \bar{t} t} h^{o} \bar{t} t \\
& +\Delta \lambda^{H^{o} \bar{b} b} H^{o} \bar{b} b+\Delta \lambda^{H^{o} \bar{t} t} H^{o} \bar{t} t \\
& +\Delta \lambda^{A^{o} \bar{b} b} A^{o} \bar{b} b+\Delta \lambda^{A^{o} \bar{t} t} A^{o} \bar{t} t \\
& +\Delta \lambda^{H^{+} \bar{t}_{L} b_{R}}\left(H^{+} \bar{t}_{L} b_{R}+\text { H.c. }\right) \\
& +\Delta \lambda^{H^{+} \bar{t}_{R} b_{L}}\left(H^{+} \bar{t}_{R} b_{L}+\text { H.c. }\right)
\end{aligned}
$$

where

$$
\begin{aligned}
& \Delta \lambda^{h^{o} \bar{b} b} \equiv \frac{g m_{b} \sin \alpha}{2 m_{W} \cos \beta}\left[-\frac{\alpha_{s}}{3 \pi} \frac{M_{\tilde{g}} \mu}{M_{S U S Y}^{2}}(\tan \beta+\cot \alpha)\right] \\
& \Delta \lambda^{h^{o} t} \equiv-\frac{g m_{t} \cos \alpha}{2 m_{W} \sin \beta}\left[-\frac{\alpha_{s}}{3 \pi} \frac{M_{\tilde{g}} \mu}{M_{S U S Y}^{2}}(\cot \beta+\tan \alpha)\right] \\
& \Delta \lambda^{H^{o} \bar{b} b} \equiv-\frac{g m_{b} \cos \alpha}{2 m_{W} \cos \beta}\left[-\frac{\alpha_{s}}{3 \pi} \frac{M_{\tilde{g}} \mu}{M_{S U S Y}^{2}}(\tan \beta-\tan \alpha)\right]
\end{aligned}
$$

$$
\begin{aligned}
& \Delta \lambda^{H^{o} t} \equiv-\frac{g m_{t} \sin \alpha}{2 m_{W} \sin \beta}\left[-\frac{\alpha_{s}}{3 \pi} \frac{M_{\tilde{g}} \mu}{M_{S U S Y}^{2}}(\cot \beta-\cot \alpha)\right] \\
& \Delta \lambda^{A^{o} \bar{b} b} \equiv \frac{i g m_{b}}{2 m_{W}} \tan \beta\left[-\frac{\alpha_{s}}{3 \pi} \frac{M_{\tilde{g}} \mu}{M_{S U S Y}^{2}}(\tan \beta+\cot \beta)\right] \\
& \Delta \lambda^{A^{\sigma^{-} t}} \equiv \frac{i g m_{t}}{2 m_{W}} \cot \beta\left[-\frac{\alpha_{s}}{3 \pi} \frac{M_{\tilde{g}} \mu}{M_{S U S Y}^{2}}(\tan \beta+\cot \beta)\right]
\end{aligned}
$$

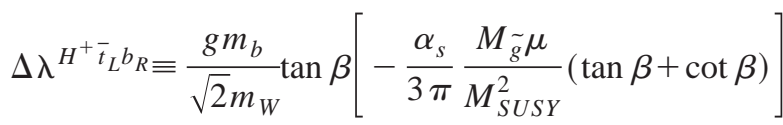

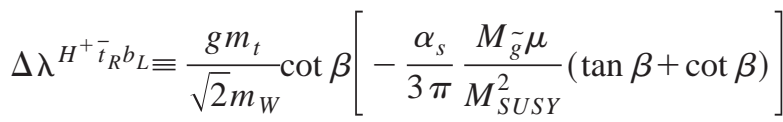

and we have omitted the primes in the quark fields and masses for brevity. The previous result in Eqs. (44), (45) is an explicit demonstration of non-decoupling of heavy squarks and gluinos in the low energy Yukawa Higgs-quarkquark interactions.

\section{DISCUSSION AND CONCLUSIONS}

The new Yukawa couplings that have been presented in Eqs. (44), (45) summarize all the non-decoupling SUSYQCD effects, to one-loop and $\mathcal{O}\left(\alpha_{s}\right)$ from nearly degenerate heavy squarks and gluinos, in the low energy Higgs-quarkquark interactions. This non-decoupling behavior can be seen in that the new low energy effective interactions do remain even in the extremely heavy SUSY masses limit, $M_{\tilde{q}} \sim M_{\tilde{g}}$ $\sim \mu \sim M_{S U S Y} \gg m_{E W}$. Furthermore, by comparison of our result with the Lagrangian for Higgs-quark-quark interactions in a general $2 \mathrm{HDM}$ we realize that the complete set of Yukawa interactions are exactly those of a 2HDMIII with the particular values of the Yukawa couplings given by Eqs. (9), 
(44) and (45). Thus, as announced, one starts in the MSSM at tree level with a Higgs sector of type 2HDMII and, after integration of squarks and gluinos, one ends up with an unconstrained 2HDMIII. The track of SUSY has not been lost but it is present in the low energy 2HDMIII interactions via the specific values of the Yukawa couplings, which in turn depend on the underlying MSSM parameters.

Notice that all the previous results are valid for any value of the input parameters in the MSSM Higgs sector, $m_{A^{o}}$ and $\tan \beta$. There are two particularly interesting situations that are worth mentioning: the large $m_{A^{o}} \gg m_{E W}$ limit, defined as the decoupling limit in [24], and the large $\tan \beta \gg 1$ limit. In the former limit, $\cot \alpha$ approaches $-\tan \beta$ (and, correspondingly, $\tan \alpha \rightarrow-\cot \beta$ ) and, as can be seen in Eq. (45), the SUSY-QCD contributions decouple in $\Delta \lambda^{h^{o} b b}$ and $\Delta \lambda^{h^{o} t t}$ and, therefore, the $h^{o}$ Yukawa couplings to quarks cannot be distinguished from the Higgs-quark-quark couplings in the $\mathrm{SM}$. The other new couplings get exactly the same nondecoupling factor, being proportional to $(\tan \beta+\cot \beta)$. However, this non-decoupling behavior is not expected to leave observable signals since, in this limit, the masses of $H^{o}, A^{o}$ and $H^{ \pm}$also get very heavy, $m_{H^{o}} \simeq m_{H^{ \pm}} \simeq m_{A^{o}}$ $\gg m_{E W}$ and they will probably not be reachable in the forthcoming colliders. The phenomenology of the MSSM Higgs sector will be just as in the SM, with $h^{o}$ being undistinguishable from the SM Higgs boson.

On the other hand, in the large $\tan \beta$ limit (with $m_{A^{o}}$ fixed) there are some corrections $\Delta \lambda / \lambda_{0}$ that grow linearly with $\tan \beta$ and can be numerically large for large values of $\tan \beta$. As can be seen in Eq. (45), these corrections, which are enhanced by $\tan \beta$ factors, are just present in $\Delta \lambda^{h^{o} \bar{b} b}, \Delta \lambda^{H^{o} \bar{b} b}, \Delta \lambda^{A^{o} \bar{b} b}, \Delta \lambda^{A^{o} \bar{t}}$ and $\Delta \lambda^{H^{+} \bar{t} b}$, in agreement with previous partial results $[11,15,12-14,16,17]$.

The results presented in this work are relevant for their phenomenological implications in Higgs production and decays at present and future colliders. Particularly interesting are the modifications they imply on the predictions for the Higgs bosons and top quark widths. By simply replacing the tree-level Yukawa couplings $\lambda_{0}^{H \bar{q} q^{\prime}} \quad\left(\lambda_{0}^{h^{0} \bar{b} b}\right.$ $=g m_{b} \sin \alpha / 2 m_{W} \cos \beta$, etc.) by the corresponding effective couplings, $\lambda_{e f f}^{H^{\bar{q} q q^{\prime}}} \equiv \lambda_{0}^{H \bar{q} q^{\prime}}+\Delta \lambda^{H \bar{q} q^{\prime}}$, with $\Delta \lambda^{H_{\bar{q}}^{\bar{q} q^{\prime}}}$ given in Eq. (45), we find out the following results for the one-loop, $\mathcal{O}\left(\alpha_{s}\right)$ SUSY-QCD contributions to the partial decay widths:

$$
\begin{aligned}
\Gamma\left(h^{0} \rightarrow \bar{b} b\right)= & \Gamma_{0}\left(h^{0} \rightarrow \bar{b} b\right) \\
& \times\left[1-\frac{2 \alpha_{s}}{3 \pi} \frac{M_{\tilde{g}} \mu}{M_{\text {SUSY }}^{2}}(\tan \beta+\cot \alpha)\right] \\
\Gamma\left(H^{0} \rightarrow \bar{b} b\right)= & \Gamma_{0}\left(H^{0} \rightarrow \bar{b} b\right) \\
& \times\left[1-\frac{2 \alpha_{s}}{3 \pi} \frac{M_{\tilde{g}} \mu}{M_{\text {SUSY }}^{2}}(\tan \beta-\tan \alpha)\right]
\end{aligned}
$$

$$
\begin{aligned}
\Gamma\left(H^{0} \rightarrow \bar{t} t\right)= & \Gamma_{0}\left(H^{0} \rightarrow \bar{t} t\right) \\
& \times\left[1-\frac{2 \alpha_{s}}{3 \pi} \frac{M_{\tilde{g}} \mu}{M_{S U S Y}^{2}}(\cot \beta-\cot \alpha)\right] \\
\Gamma\left(A^{0} \rightarrow \bar{b} b\right)= & \Gamma_{0}\left(A^{0} \rightarrow \bar{b} b\right) \\
& \times\left[1-\frac{2 \alpha_{s}}{3 \pi} \frac{M_{\tilde{g}} \mu}{M_{S U S Y}^{2}}(\tan \beta+\cot \beta)\right] \\
\Gamma\left(A^{0} \rightarrow \bar{t} t\right)= & \Gamma_{0}\left(A^{0} \rightarrow \bar{t} t\right) \\
& \times\left[1-\frac{2 \alpha_{s}}{3 \pi} \frac{M_{\tilde{g}} \mu}{M_{S U S Y}^{2}}(\tan \beta+\cot \beta)\right] \\
\Gamma\left(H^{+} \rightarrow t \bar{b}\right)= & \Gamma_{0}\left(H^{+} \rightarrow t \bar{b}\right) \\
& \times\left[1-\frac{2 \alpha_{s}}{3 \pi} \frac{M_{\tilde{g}} \mu}{M_{S U S Y}^{2}}(\tan \beta+\cot \beta)\right] \\
& \times\left[1-\frac{2 \alpha_{s}}{3 \pi} \frac{M_{\tilde{g}} \mu}{M_{S U S Y}^{2}}(\tan \beta+\cot \beta)\right] \\
\Gamma\left(t \rightarrow H^{+} b\right)= & \Gamma_{0}\left(t \rightarrow H^{+} b\right) \\
& {[1] }
\end{aligned}
$$

where $\Gamma_{0}$ are the tree-level widths. These formulas are for nearly degenerate heavy squarks and gluinos and apply for any value of $m_{A^{o}}$ and $\tan \beta$. They are in perfect agreement with the expressions obtained from a complete diagrammatic computation of the partial widths (see, for instance, $[16,17]$ for the $h^{o}$ and $H^{+}$cases). Notice that, in most of the MSSM parameter space, these SUSY-QCD corrections all have the same sign and universal character, specially in the large $\tan \beta$ regime, and they will be very useful in a global experimental analysis, because they will yield strongly correlated signals in event rates from Higgs (and top) decays [23]. These corrections can be used for indirect but very efficient SUSY searches at the present and future colliders [11-17,19-23].

In summary, in this work we have computed the SUSYQCD contributions to all the Higgs-quark-quark couplings at the one-loop level and $\mathcal{O}\left(\alpha_{s}\right)$ in the limit of very heavy squarks and gluinos. We have found that, after integrating out the squark and gluino fields, these contributions do not decouple in the Higgs-quark-quark interactions, and give as a result a low energy effective theory of type 2HDMIII, with specific values for the couplings given in terms of the underlying MSSM parameters.

\section{ACKNOWLEDGMENTS}

This work has been supported in part by the Spanish Ministerio de Ciencia y Tecnología under projects CICYT FPA 2000-0980, CICYT AEN 97-1693 and PB98-0782. 
APPENDIX A: HIGGS-SQUARK-SQUARK INTERACTION LAGRANGIAN

The interactions between Higgs particles and squarks, in the chiral squark basis, are described by the following interaction Lagrangian:

$$
\mathcal{L}(H, \tilde{q})=\sum_{H} H \widetilde{Q}_{c h}^{\dagger} A_{H} \widetilde{Q}_{c h}
$$

where the $H$ index runs along $H=h^{o}, H^{o}, A^{o}, H^{1}, H^{2}$. In this appendix we give the expressions for the coupling $4 \times 4$ matrices $A_{H}$ corresponding to the different Higgs fields.

For $h^{o}$,

$$
A_{h^{o}}=\left(\begin{array}{cc}
a_{h^{o}}^{\tilde{t}} & 0 \\
0 & a_{h^{o}}^{\tilde{b}}
\end{array}\right)
$$

where

$$
\begin{aligned}
& a_{h^{o}}^{\tilde{t}}=\left(\begin{array}{cc}
\frac{g m_{Z}}{c_{W}}\left(\frac{1}{2}-\frac{2}{3} s_{W}^{2}\right) \sin (\alpha+\beta)-\frac{g m_{t}^{2} \cos \alpha}{m_{W} \sin \beta} & -\frac{g m_{t}}{2 m_{W} \sin \beta}\left(\mu \sin \alpha+A_{t} \cos \alpha\right) \\
-\frac{g m_{t}}{2 m_{W} \sin \beta}\left(\mu \sin \alpha+A_{t} \cos \alpha\right) & \frac{g m_{Z}}{c_{W}} \frac{2}{3} s_{W}^{2} \sin (\alpha+\beta)-\frac{g m_{t}^{2} \cos \alpha}{m_{W} \sin \beta}
\end{array}\right) \\
& a_{h^{o}}^{\tilde{b}}=\left(\begin{array}{cc}
\frac{g m_{Z}}{c_{W}}\left(-\frac{1}{2}+\frac{1}{3} s_{W}^{2}\right) \sin (\alpha+\beta)+\frac{g m_{b}^{2} \sin \alpha}{m_{W} \cos \beta} & \frac{g m_{b}}{2 m_{W} \cos \beta}\left(\mu \cos \alpha+A_{b} \sin \alpha\right) \\
\frac{g m_{b}}{2 m_{W} \cos \beta}\left(\mu \cos \alpha+A_{b} \sin \alpha\right) & -\frac{g m_{Z}}{c_{W}} \frac{1}{3} s_{W}^{2} \sin (\alpha+\beta)+\frac{g m_{b}^{2} \sin \alpha}{m_{W} \cos \beta}
\end{array}\right) .
\end{aligned}
$$

For $H^{o}$,

$$
A_{H^{o}}=\left(\begin{array}{cc}
a_{H^{o}}^{\tilde{t}} & 0 \\
0 & a_{H^{o}}^{\tilde{b}}
\end{array}\right)
$$

where

$$
\begin{aligned}
& a_{H^{o}}^{\tilde{t}}=\left(\begin{array}{cc}
-\frac{g m_{Z}}{c_{W}}\left(\frac{1}{2}-\frac{2}{3} s_{W}^{2}\right) \cos (\alpha+\beta)-\frac{g m_{t}^{2} \sin \alpha}{m_{W} \sin \beta} & -\frac{g m_{t}}{2 m_{W} \sin \beta}\left(-\mu \cos \alpha+A_{t} \sin \alpha\right) \\
-\frac{g m_{t}}{2 m_{W} \sin \beta}\left(-\mu \cos \alpha+A_{t} \sin \alpha\right) & -\frac{g m_{Z}}{c_{W}} \frac{2}{3} s_{W}^{2} \cos (\alpha+\beta)-\frac{g m_{t}^{2} \sin \alpha}{m_{W} \sin \beta}
\end{array}\right) \\
& a_{H^{o}}^{\tilde{b}}=\left(\begin{array}{cc}
-\frac{g m_{Z}}{c_{W}}\left(-\frac{1}{2}+\frac{1}{3} s_{W}^{2}\right) \cos (\alpha+\beta)-\frac{g m_{b}^{2} \cos \alpha}{m_{W} \cos \beta} & -\frac{g m_{b}}{2 m_{W} \cos \beta}\left(-\mu \sin \alpha+A_{b} \cos \alpha\right) \\
-\frac{g m_{b}}{2 m_{W} \cos \beta}\left(-\mu \sin \alpha+A_{b} \cos \alpha\right) & \frac{g m_{Z}}{c_{W}} \frac{1}{3} s_{W}^{2} \cos (\alpha+\beta)-\frac{g m_{b}^{2} \cos \alpha}{m_{W} \cos \beta}
\end{array}\right) .
\end{aligned}
$$

For $A^{o}$, 


$$
A_{A^{o}}=\left(\begin{array}{cc}
a_{A^{o}}^{\tilde{t}} & 0 \\
0 & a_{A^{o}}^{\tilde{b}}
\end{array}\right)
$$

where

$$
\begin{aligned}
& a_{A^{o}}^{\tilde{t}}=\left(\begin{array}{cc}
0 & \frac{i g m_{t}}{2 m_{W}}\left(\mu+A_{t} \cot \beta\right) \\
-\frac{i g m_{t}}{2 m_{W}}\left(\mu+A_{t} \cot \beta\right) & 0
\end{array}\right) \\
& a_{A^{o}}^{\tilde{b}}=\left(\begin{array}{cc}
\frac{i g m_{b}}{2 m_{W}}\left(\mu+A_{b} \tan \beta\right) \\
0 & 0
\end{array}\right) .
\end{aligned}
$$

For $H^{1}$ and $H^{2}$,

$$
A_{H^{1}}=\left(\begin{array}{cc}
0 & a_{H^{1}} \\
a_{H^{1}}^{T} & 0
\end{array}\right), \quad A_{H^{2}}=\left(\begin{array}{cc}
0 & a_{H^{2}} \\
-a_{H^{2}}^{T} & 0
\end{array}\right)
$$

where

$$
\begin{aligned}
& a_{H^{1}}=\left(\begin{array}{cc}
\frac{g}{2 m_{W}}\left(m_{b}^{2} \tan \beta+m_{t}^{2} \cot \beta-m_{W}^{2} \sin 2 \beta\right) & \frac{g m_{b}}{2 m_{W}}\left(\mu+A_{b} \tan \beta\right) \\
\frac{g m_{t}}{2 m_{W}}\left(\mu+A_{t} \cot \beta\right) & \frac{g m_{t} m_{b}}{2 m_{W}}(\tan \beta+\cot \beta)
\end{array}\right) \\
& a_{H^{2}}=i a_{H^{1}} .
\end{aligned}
$$

\section{APPENDIX B: LARGE MASS EXPANSION OF LOOP INTEGRALS AND MIXING ANGLES}

Here we give the expansions of the loop integrals and mixing angles in inverse powers of the SUSY mass parameters. For the two- and three-point integrals that appear in Eqs. (34) and (38), we follow the definitions and conventions of [26]. The integrals are performed in $D=4-\epsilon$ dimensions and the divergent contributions are regularized by $\Delta \equiv(2 / \epsilon)$ $-\gamma_{E}+\log (4 \pi)-\log \left(M_{S U S Y}^{2} / \mu_{0}^{2}\right)$, with the corresponding inclusion of the energy scale given here by $\mu_{0}$.

The two-point integrals are given by

$$
\begin{gathered}
\mu_{0}^{4-D} \int \frac{d^{D} k}{(2 \pi)^{D}} \frac{\left\{1 ; k^{\mu}\right\}}{\left[k^{2}-m_{1}^{2}\right]\left[(k+q)^{2}-m_{2}^{2}\right]} \\
=\frac{i}{16 \pi^{2}}\left\{B_{0} ; q^{\mu} B_{1}\right\}\left(q^{2} ; m_{1}^{2}, m_{2}^{2}\right) .
\end{gathered}
$$

The three-point integrals are given by

$$
\begin{aligned}
\mu_{0}^{4-D} & \int \frac{d^{D} k}{(2 \pi)^{D}} \\
& \times \frac{\left\{1 ; k^{\mu}\right\}}{\left[k^{2}-m_{1}^{2}\right]\left[\left(k+p_{1}\right)^{2}-m_{2}^{2}\right]\left[\left(k+p_{1}+p_{2}\right)^{2}-m_{3}^{2}\right]} \\
= & \frac{i}{16 \pi^{2}}\left\{C_{0} ; p_{1}^{\mu} C_{11}+p_{2}^{\mu} C_{12}\right\}\left(p_{1}^{2}, p_{2}^{2}, p^{2} ; m_{1}^{2}, m_{2}^{2}, m_{3}^{2}\right),
\end{aligned}
$$

where $p=-p_{1}-p_{2}$.

We are considering here the limit where all the squarks and gluinos are nearly degenerate and very heavy as compared to the electroweak scale. In terms of the MSSM parameters, this limit is reached by choosing $M_{S U S Y} \sim M_{\tilde{g}}$ $\sim M_{\tilde{Q}} \sim M_{\tilde{U}} \sim M_{\tilde{D}} \sim \mu \gg m_{E W}$, and the physical squark masses can be written as $M_{\tilde{q}_{1,2}}^{2}=M_{S U S Y}^{2} \pm m_{q} X_{q}$. The results of the large $M_{S U S Y}$ expansions of the loop integrals in this limit are as follows: 


$$
\begin{aligned}
& C_{0}\left(p_{q}^{2}, p_{H}^{2}, p_{q^{\prime}}^{2} ; M_{\tilde{g}}^{2}, M_{\tilde{q}_{i}}^{2}, M_{\tilde{q}_{j}}^{2}\right) \simeq-\frac{1}{2 M_{S U S Y}^{2}}+\mathcal{O}\left(\frac{m_{E W}}{M_{S U S Y}^{3}}\right) \\
& C_{11}\left(p_{q}^{2}, p_{H}^{2}, p_{q^{\prime}}^{2} ; M_{\tilde{g}}^{2}, M_{\tilde{q}_{i}}^{2}, M_{\tilde{q}_{j}}^{2}\right) \simeq \frac{1}{3 M_{S U S Y}^{2}}+\mathcal{O}\left(\frac{m_{E W}}{M_{S U S Y}^{3}}\right) \\
& C_{12}\left(p_{q}^{2}, p_{H}^{2}, p_{q^{\prime}}^{2} ; M_{\tilde{g}}^{2}, M_{\tilde{q}_{i}}^{2}, M_{\tilde{q}_{j}}^{2}\right) \simeq \frac{1}{6 M_{S U S Y}^{2}}+\mathcal{O}\left(\frac{m_{E W}}{M_{S U S Y}^{3}}\right) \\
& B_{0}\left(p_{q}^{2} ; M_{\tilde{g}}^{2}, M_{\tilde{q}_{i}}^{2}\right) \simeq \Delta+(-1)^{i} \frac{m_{q} X_{q}}{2 M_{S U S Y}^{2}}+\mathcal{O}\left(\frac{m_{E W}^{2}}{M_{S U S Y}^{2}}\right) \\
& B_{1}\left(p_{q}^{2} ; M_{\tilde{g}}^{2}, M_{\tilde{q}_{i}}^{2}\right) \simeq-\frac{1}{2} \Delta-(-1)^{i} \frac{m_{q} X_{q}}{3 M_{S U S Y}^{2}}+\mathcal{O}\left(\frac{m_{E W}^{2}}{M_{S U S Y}^{2}}\right)
\end{aligned}
$$

where $X_{q}$ are defined in Eq. (3), $p_{q}, p_{q^{\prime}}$ and $p_{H}$ are the external momenta of quarks and Higgs particles, respectively, and $m_{E W}$ refers generically to any external momentum or mass of the order of the electroweak scale.
The expansions for the mixing angles in the large $M_{S U S Y}$ limit are as follows:

$$
\begin{aligned}
\sin 2 \theta_{q} & \simeq 1+\mathcal{O}\left(\frac{m_{E W}^{2}}{M_{S U S Y}^{2}}\right), \\
\cos 2 \theta_{q} & \simeq 0+\mathcal{O}\left(\frac{m_{E W}}{M_{S U S Y}}\right),
\end{aligned}
$$

thus, our choice of large MSSM parameters above implies nearly maximal squark mixing.

Finally, notice that we have written explicitly in Eqs. (B3) and (B4) only the leading terms in the expansions. The next to leading terms of these expansions can be found in $[16,17]$. In particular, because of our choice of $\mu \sim M_{S U S Y}, X_{q}$ are of $\mathcal{O}\left(M_{S U S Y}\right)$ and, therefore, the second terms in the expansions of $B_{0}$ and $B_{1}$ are of $\mathcal{O}\left(m_{E W} / M_{S U S Y}\right)$. For the $B_{0}$ case, this term will also contribute, via Eq. (34) to the nondecoupling effects in the quark propagators. Regarding the three-point functions, it is $C_{0}$ that via Eq. (38) will contribute to the non-decoupling terms.
[1] For an updated compilation of works, see Proceedings for 5th International Symposium on Radiative Corrections (RADCOR-2000), Carmel, California, 2000. Slides available at http://radcor2000.slac.stanford.edu/program.html

[2] H. E. Haber and G. L. Kane, Phys. Rep. 117, 75 (1985).

[3] J. F. Gunion and H. E. Haber, Nucl. Phys. B272, 1 (1986); B278, 449 (1986); B402, 567(E) (1993); J. F. Gunion, H. E. Haber, G. Kane, and S. Dawson, The Higgs Hunter's Guide (Addison-Wesley, Reading, MA, 1990).

[4] A. Dabelstein, Nucl. Phys. B456, 25 (1995).

[5] J. Guasch, R. A. Jiménez, and J. Solà, Phys. Lett. B 360, 47 (1995).

[6] R. A. Jiménez and J. Solà, Phys. Lett. B 389, 53 (1996).

[7] J. A. Coarasa, R. A. Jiménez, and J. Solà, Phys. Lett. B 389, 312 (1996).

[8] A. Bartl, H. Eberl, K. Hidaka, T. Kon, W. Majerotto, and Y. Yamada, Phys. Lett. B 378, 167 (1996).

[9] A. Dobado, M. J. Herrero, and S. Peñaranda, Eur. Phys. J. C 7, 313 (1999); in Barcelona 1997, Quantum Effects in the Minimal Supersymmetric Standard Model, edited by J. Solà (World Scientific, Singapore, 1998), p. 266-286, hep-ph/9711411; Eur. Phys. J. C 12, 673 (2000); 17, 487 (2000).

[10] T. Appelquist and J. Carazzone, Phys. Rev. D 11, 2856 (1975).

[11] M. Carena, S. Mrenna, and C. E. M. Wagner, Phys. Rev. D 60, 075010 (1999); 62, 055008 (2000).

[12] K. S. Babu and C. Kolda, Phys. Lett. B 451, 77 (1999).

[13] M. Carena, D. Garcia, U. Nierste, and C. E. Wagner, Nucl. Phys. B577, 88 (2000).

[14] H. Eberl, K. Hidaka, S. Kraml, W. Majerotto, and Y. Yamada, Phys. Rev. D 62, 055006 (2000).

[15] F. Borzumati, G. R. Farrar, N. Polonsky, and S. Thomas, Nucl. Phys. B555, 53 (1999).
[16] H. E. Haber, M. J. Herrero, H. E. Logan, S. Peñaranda, S. Rigolin, and D. Temes, Phys. Rev. D 63, 055004 (2001).

[17] M. J. Herrero, S. Peñaranda, and D. Temes, Phys. Rev. D 64, 115003 (2001).

[18] D. Atwood, L. Reina, and A. Soni, Phys. Rev. D 55, 3156 (1997) and references therein.

[19] H. E. Haber, M. J. Herrero, H. E. Logan, S. Peñaranda, S. Rigolin, and D. Temes, hep-ph/0102169; invited talk given by M. J. Herrero at the RADCOR-2000 symposium, Carmel, California, 2000. Slides available at http:// radcor2000.slac.stanford.edu/program.html

[20] M. Carena, H. E. Haber, H. E. Logan, and S. Mrenna, Phys. Rev. D 65, 055005 (2002).

[21] M. J. Herrero, invited talk at the XXIX International Meeting on Fundamental Physics, Sitges, Barcelona, Spain, 2001, to appear in the Proceedings, FTUAM/01-11. Slides available at http://29imfp.gae.ucm.es/sp.php3

[22] J. Guasch, W. Hollik, and S. Peñaranda, Phys. Lett. B 515, 367 (2001).

[23] A. M. Curiel, M. J. Herrero, D. Temes, and J. F. de Troconiz, Phys. Rev. D 65, 075006 (2002).

[24] H. E. Haber and Y. Nir, Nucl. Phys. B335, 363 (1990); H. E. Haber, in Proceedings of the U.S.-Polish Workshop, Warsaw, Poland, 1994, edited by P. Nath, T. Taylor, and S. Pokorski (World Scientific, Singapore, 1995), pp. 49-63.

[25] H. E. Haber and R. Hempfling, Phys. Rev. Lett. 66, 1815 (1991); Phys. Rev. D 48, 4280 (1993); Y. Okada, M. Yamaguchi, and T. Yanagida, Prog. Theor. Phys. 85, 1 (1991); Phys. Lett. B 262, 54 (1991); J. Ellis, G. Ridolfi, and F. Zwirner, ibid. 257, 83 (1991); 262, 477 (1991); R. Barbieri and M. Frigeni, ibid. 258, 167 (1991); 258, 395 (1991); for an updated 
study see M. Carena, H. E. Haber, S. Heinemeyer, W. Hollik, C. E. M. Wagner, and G. Weiglein, Nucl. Phys. B580, 29 (2000); J. R. Espinosa and R.-J. Zhang, J. High Energy Phys. 03, 026 (2000); Nucl. Phys. B586, 3 (2000), and references therein.

[26] W. Hollik, in Precision Tests of the Standard Electroweak Model, edited by P. Langacker (World Scientific, Singapore, 1995), pp. 37-116. 